\title{
Non-visible penetrating brain trauma: a case report
}

\author{
Rosanna Varutti ${ }^{*}$, Alessandro Mosca, Nicola Latronico \\ Department of Anesthesia, Critical Care and Emergency, Spedali Civili University Hospital, Brescia, Italy
}

\author{
Received: 12 October 2019 \\ Accepted: 5 November 2019 \\ Published online: 13 November \\ 2019 \\ *Corresponding author: Rosanna \\ Varutti, rosanna.varutti@gmail.com \\ Competing interests: None. \\ Funding information: None \\ Citation: Varutti R, Mosca A, \\ Latronico N. Non-visible penetrating \\ brain trauma: a case report. Journal \\ of Emergency Practice and Trauma \\ 2020; 6(1): 50-52. doi: 10.15171/ \\ jept.2019.20
}

\begin{abstract}
Objective: One of the most frequent causes of death and acquired disability in the pediatric population is the Traumatic Brain Injury (TBI). TBI is secondary to falls, road traffic and vehicle collisions, child abuse and assaults. Penetrating brain injury is a severe form of traumatic brain injury. Blunt head injury is more frequent than TBI in children, but the second one carries a poor prognosis and a worse outcomes.

Case Presentation: We report a pediatric case of penetrating craniofacial trauma, caused by a pencil in to the eye, and the successful of multidisciplinary management.

Conclusion: Traumatic head injuries in children are frequently seen in the emergency ward, but the penetrating head injuries are unusual in young children. This case shows the importance of the 'first golden hour' after head trauma. The collaboration between team members, added to a promptly and invasive strategy, allowed to reduce neurological sequelae.

Keywords: Pediatric, Penetrating, Brain, Trauma
\end{abstract}

\section{Introduction}

One of the most frequent causes of death and acquired disability in the pediatric population is the traumatic brain injury (TBI). TBI is secondary to falls, road traffic and vehicle collisions, child abuse and assaults. Penetrating brain injury is a severe form of TBI. Blunt head injury is more frequent than TBI in children, but the second one carries a poor prognosis and worse outcomes (1).

In the pre-hospital setting, a penetrating intracranial foreign body is a typical situation in which a "scoop and run" is recommended because a surgical approach is needed for its removal and an intensive perioperative management is particularly complex (2).

Hemiparesis and cranial nerve deficits are the most common neurological abnormalities observed in those situations; immediate seizures may be present $(1,2)$. We report a pediatric case of penetrating craniofacial trauma caused by a pencil into the eye, and the successful multidisciplinary management.

\section{Case Presentation}

A 7-year-old girl was admitted to the casualty of Civili Hospital in Brescia for a penetrating craniofacial trauma. Her father expressed an accidental fall while walking with a pencil hold in her hands. The crying child was transferred to a local hospital and the first clinical evaluation revealed only a small wound $(<1 \mathrm{~cm})$ at the left inferior palpebral, and an anisocoria with associated visual deficit. The anisocoria and the visual deficit of her left eye were suggestive for a direct eye trauma. The computed tomography (CT) scan section showed the presence of an entire pencil that crossed from the left orbital fossa into the brain (Figure $1 \mathrm{~A}$ and $1 \mathrm{Bb}$ ). Nonsystemic neurological deficit was observed, and she was continuously monitored (heart rate, non-invasive blood pressure, peripheral oxygen saturation). The patient was promptly transferred to the closed hub hospital (Spedali Civili di Brescia). A multidisciplinary team (anaesthetists, neurosurgeons, maxillofacial surgeons, ophthalmologists and radiologists) performed the first clinical evaluation, and they planned the radiological examination as well as the subsequent treatment and potential complications. The CT scan showed a foreign body nearest to the left internal carotid artery; therefore an angiography examination was needed.

After a neurological evaluation, the patient was intubated in order to facilitate other diagnosis paths and treatment. She was invasively monitored using a venous, arterial, vesical catheter and a temperature monitoring probe.

A selective angiography was made (both internal carotid arteries and left vertebral artery were studied) and it showed an occlusion of the intracranial tract of the left internal carotid artery at the lecherous part because of pencil compression. The compensation of the left cerebral perfusion through anterior and left posterior communicating arteries was confirmed and the left internal 


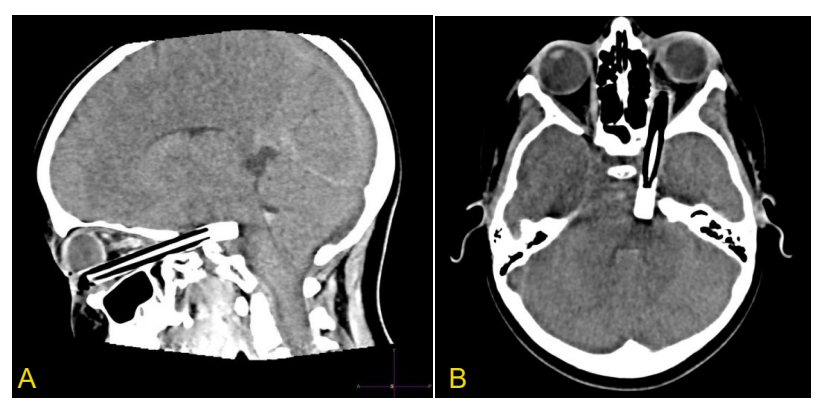

Figure 1. Computed Tomography (CT) scan showing the presence of an entire pencil that crossed from the left orbital fossa into the brain.

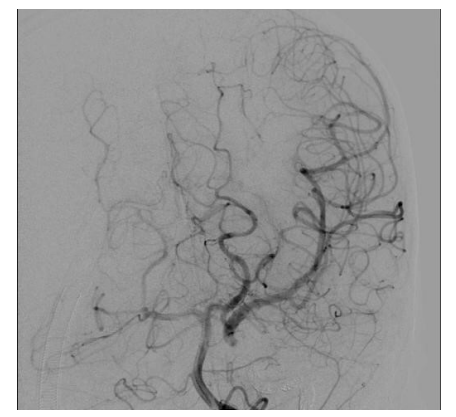

Figure 2. A selective angiography showing an occlusion of the intracranial tract of the left internal carotid artery at the lecherous part due to pencil compression.

carotid artery was embolized. At the end of the procedure, a final angiography confirmed a complete occlusion of the left internal carotid artery with normal vascularization of left brain parenchyma (Figure 2). During the procedure (about 1 hour), the patient was sedated with propofol and she was hemodynamically stable.

The patient was moved to the neurosurgical operating room for a combined second step surgery (neurosurgery and maxillofacial surgery). The target was to isolate vascular and cerebral structures before the removal of penetrated object.

A left pterional craniotomy access was made by neurosurgeons obtaining a dissection of the Silvanus's fissure, the exposure of the left internal carotid artery, left posterior communicating artery and all the nearest vascular structures. A subarachnoid haemorrhage at the interpeduncolate cisterna was observed. Subsequently, the maxillofacial surgeons safely removed the foreign body from the cutaneous wound at the left inferior palpebral with a little bleeding but neither transfusion nor catecholamines was needed. She was hemodynamically stable during the surgery and normothermic.

The young girl was transferred to the PICU (pediatric intensive care unit), where she was extubated for 12 hours. When awake, no neurological deficits were recorded, except for a loss of her left eye visus. The ICU stay was uneventful she was discharged to the pediatric maxillofacial ward 2 days later.
A quick physical rehabilitation was started during recovery and a broad antibiotic therapy was started to avoid cerebral infection. Post-operative ophthalmologist and neurosurgical evaluations were continuously performed. The patient and her parents were supported by a psychologist to understand and accept the visual deficit. A good clinical course was reported during the recovery. At the fourteenth day, the patient was discharged home.

\section{Discussion}

The age of the patient, the complexity and rarity of the traumatism, and the need to intervene promptly in an emergency regime are important factors in determining the clinical therapeutic management. Head trauma is common in children, often of mild to moderate entity and most of them are due to falls. Conversely, a penetrating brain trauma caused by bullets, foreign bodies and shrapnel is extremely rare in childhood (1-4).

The mechanism of injury is different from low velocity head injuries (hemorrhage) to gunshot and missile injuries (cavitations and necrotic concentric zones) (5).

In this case an operative angiography was made to avoid massive hemorrhage from internal carotid after the pencil removal and to evade cerebral embolisms. The artery was obstructed and injured by the foreign body. The risks of ischemia of the left cerebrum were real, but the collateral flux demonstrated during the angiography had guaranteed a good residual cerebral perfusion. Neurosurgical procedure was difficult and the risk of blooding and lesion of important cerebral structures were real.

The prognosis is generally favorable if the vital centers and large vessels are spared from injury; instead, the early treatment reduces the risk of delayed infections and vascular and epileptic complications. The young patient was rapidly extubated for a neurological evaluation and continuous monitoring and she was discharged home without major complications (except for the left visual damage).

\section{Conclusion}

Traumatic head injuries in children are frequently seen in the emergency ward, but the penetrating head injuries are unusual in young children.

This case shows the importance of the 'first golden hour' after head trauma. The collaboration between team members, added to a promptly and invasive strategy, allowed to reduce neurological sequelae.

\section{Authors' contributions}

Each author contributed equally to study design, drafting article, reading critically and accepted finally proof.

\section{Ethical issues}

Informed consent was obtained from the parents' patient for publication of this report. 


\section{References}

1. Karim T, Topno M. An unusual case of penetrating head injury in a child. J Emerg Trauma Shock 2010; 3(2): 197-8. doi: 10.4103/0974-2700.62113.

2. Atabaki SM. Pediatric head injury. Pediatr Rev 2007; 28(6): 215-24. doi: 10.1542/pir.28-6-215.

3. Rasmussen LK. Neurocritical care for severe pediatric traumatic brain injury. Medscape; Updated: May 30, 2018. https://emedicine.medscape.com/article/909105- overview\#a1.

4. Drosos E, Giakoumettis D, Blionas A, Mitsios A, Sfakianos G, Themistocleous M. Pediatric nonmissile penetrating head injury: case series and literature review. World Neurosurg 2018; 110: 193-205. doi: 10.1016/j.wneu.2017.11.037.

5. Mikhael M, Frost E, Cristancho M. Perioperative care for pediatric patients with penetrating brain injury: a review. J Neurosurg Anesthesiol 2018; 30(4): 290-8. doi: 10.1097/ ana.0000000000000441. 